See discussions, stats, and author profiles for this publication at: https://www.researchgate.net/publication/325483728

\title{
Modelling the night sky brightness and light pollution sources of Montsec protected area
}

Article in Journal of Quantitative Spectroscopy and Radiative Transfer · June 2018

CITATIONS

0

4 authors, including:

Martin Aubé

Cégep de Sherbrooke

50 PUBLICATIONS 405 CITATIONS

SEE PROFILE

Manuel Garcia

Universitat Politècnica de Catalunya

19 PUBLICATIONS 31 CITATIONS

SEE PROFILE

Some of the authors of this publication are also working on these related projects:

Red española de estudios sobre la contaminación lumínica View project

Cities at Night View project
READS

98

Salvador J. Ribas

Parc Astronòmic Montsec

29 PUBLICATIONS 81 CITATIONS

SEE PROFILE 


\title{
Modelling the night sky brightness and light pollution sources of Montsec protected area
}

\author{
Hector Linares $^{\mathrm{a}}$, Eduard Masana ${ }^{\mathrm{a}}$, Salvador J. Ribas ${ }^{\mathrm{b}, \mathrm{a}}$, Manuel Garcia - \\ Gil $^{c}$, Francesca Figueras ${ }^{\mathrm{a}}$, Martin Aubéd \\ ${ }^{a}$ Institut de Ciències del Cosmos (ICC-UB-IEEC), Martí i Franquès, 1, E-08028 \\ Barcelona, Spain \\ ${ }^{b}$ Parc Astronòmic Montsec, Cami del coll d'Ares s/n, E-25691 Ager, Spain \\ ${ }^{c}$ Servei de Prevenció de la Contaminació Acustíca i Lumínica, Generalitat de \\ Catalunya., Av. Diagonal 523-525, E-08029, Barcelona, Spain \\ ${ }^{d}$ Département de physique, Cégep de Sherbrooke, Sherbrooke, Quebec, J1E 4K1, Canada
}

\begin{abstract}
We proceeded to the modelling of the night sky brightness of Montsec area (north-east of Spain), an astronomical protected area certified as a Starlight Reserve. We have used the hyperspectral version of ILLUMINA, an artificial sky brightness model. Ground based measurements for Montsec and other areas of Catalonia (Ribas and Canal-Domingo, 2015; Ribas, 2016), including both photometric and spectroscopic data, has been used to fit and evaluate the input parameters of the model. In this first modelling attempt, Lleida, the biggest city in the area, has been considered as the unique source of light pollution. In 2014 there was an update of the lighting infrastructure in Lleida. A detailed comparison of the sky brightness before and after the change is shown in order to measure the effects that different kind of lamps can produce. This information could be used to plan for future updates and improvements of the lighting systems in the area.
\end{abstract}

Keywords: light pollution, modelling, scattering

\section{Introduction}

Light pollution was already a noticeable problem for astronomy in the second half of the twentieth century when trying to find new locations for astronomical observatories (Riegel, 1973; Walker, 1970, 1973). 
The first attempts to derive a propagation law for artificial night-sky illumination came soon after. Bertiau et al. (1973) and Treanor (1973) presented simplified models that estimates the contribution of aerosol scattering to the zenith brightness due to light coming from nearby cities. The artificial zenith brightness was modeled by the population-brightness function $F(D)$ and the brightness-distance function $Q(D)$ of the surrounding cities. Berry (1976) modified the brightness-population function in agreement with the work of Normandin (1974) in the province of Québec, to better fit the data obtained from Southern Ontario. Walker (1977) described new $F(P)$ and $Q(D)$ using measurements from California.

A major step towards a more complete model was proposed by Garstang (1986). In Garstang's model, cities were not considered as point sources but as circular uniform surfaces; the amount of aerosols was an adjustable parameters and different scale heights for molecules and aerosols were established. It also accounted for ground reflectance and the percentage of light emitted above the horizontal with a prescribed angular emission function.

Nowadays, there are two prominent light pollution models: $\mathrm{MSNsR}_{\mathrm{Au}}$ (Kocifaj, 2007), and ILLUMINA (Aubé et al., 2005). Both models accept the heterogeneity of ground-based light sources (spectra and light output angle distribution). MSNsR $\mathrm{Au}_{\mathrm{Au}}$ takes into account first order scattering only, which is suited for optically thin atmospheres and observers relatively close to cities and for low elevation angles. ILLUMINA on the other hand includes first and second order scattering that allows experiments in any direction and distance. Taking into account second order scattering makes ILLUMINA very time consuming with respect $\mathrm{MSNsR}_{\mathrm{Au}}$. For that reason $\mathrm{MSNsR}_{\mathrm{Au}}$ is better suited for experiments involving a large number of grid point (large geographical domain with high spacial resolution). However, Aubé (2007) showed that the second-order relative contribution rises with distance from city limits and may contribute up to 66 percent of the total zenith radiance for remote sites, therefore experiments with observer's location far from cities should take into account second order scattering.

The present work is based on ILLUMINA because of the low number of lighted grid points and the large distance between the observer and the sources. Our aim is to better understand what kind of sources produces light pollution and how they affect astronomical observations. The output of ILLUMINA is converted to astronomical magnitudes to build all-sky maps comparable with the ones obtained with ground based measurements. We focus on the effect of light pollution in the Johnson-Cousins photometric 
system visual filters $B, V$ and $R$ (Johnson and Morgan, 1953, 1955; Cousins, 1976) in any line of sight from the observer.

The main goal is to evaluate the light pollution that Parc Astronòmic Montsec (PAM) is receiving from Lleida, the biggest city in the area, located approximately $50 \mathrm{~km}$ south from PAM. Montsec is an astronomical protected area in the Northeast of Spain, labeled as Reference Point accordint to Law 6/2001 of Government of Catalonia due to its pristine conditions and also certified as Starlight Reserve. This case of study is interesting not only for the relevance of the PAM but to compare the effects of two different city lighting systems. In 2014 Lleida updated its light source inventory mostly by replacing mercury vapor and some metal halide lights by LEDs.

The paper is organized as follow. In section 2 we introduce the methodology, describing briefly ILLUMINA and how its output is converted to astronomical magnitudes. In section 3 we characterize the parameters of the study: location, atmosphere, lines of sight, inventory of lights, etc. In section 4 the results are presented in the form of sky brightness all-sky maps. Finally the results are discussed in section 5 .

\section{Methodology}

The methodology used to compute sky brightness above the observer is divided in two main blocks.

The first one is based on the ILLUMINA project (Aubé et al., 2005; Aubé and Roby, 2014), an open source code that produces several outputs. We will exclusively use the sky spectral radiance output. The second block is the post-processing, which consists in the conversion of ILLUMINA spectral radiance to proper astronomic magnitudes and displaying them in all-sky maps. Such conversion will allow to proceed to comparison with images obtained by measurements on the ground.

\subsection{ILLUMINA}

ILLUMINA is a sophisticated light propagation numerical model able to simulate with precision the human induced sky radiance. It takes into account first and second order scattering both direct and after reflecting on the ground (see figure 1). Light sources, as cities and towns, are considered as combination of many circular areas each having its homogeneous spectral behavior, its light output angle distribution respect to the vertical plane, its lamp height respective to the ground and its obstacles size and distance. 


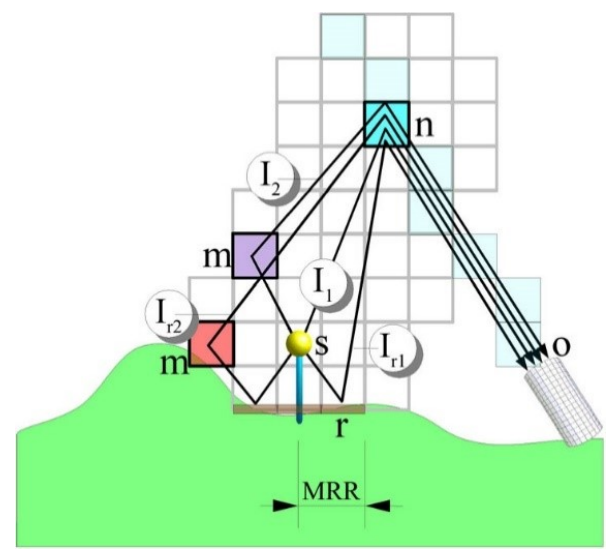

Figure 1: Scheme of the model used by ILLUMINA to compute the flux received in each direction. S, source of light; O, observer's location; n, any 3D cell in the line of sight of the observer; $\mathrm{m}$, cells that scatter light to $\mathrm{n} ; I_{1}$, first order scattering path; $I_{2}$, second order scattering path; $I_{r 1}$, reflection and first order scattering path; $I_{r 2}$, reflection and second order scattering path; r, reflection on the ground; MRR, maximum radius taken into account for the reflection. Image from Aubé et al. (2005).

These properties are computed by defining a detailed inventory of the lighting systems for each light source considered. The intensity of the light is computed in each point using satellite data and, in general, it is not uniform over the city area.

It also takes into account of the geographical characteristics of the environment (elevation and reflectivity of the ground) by using a digital elevation model and satellite spectral albedo information, it also uses multiple parameters to define the atmosphere.

To compute the sky brightness in a given direction, ILLUMINA divides the atmosphere in 3D cells and takes the sum over the cells along the line of sight ( $n$ in figure 1 ) to integrate the total light scattered towards the observer in that direction. The model assume that the total flux is mainly explained by the combination of the first-order scattering $\left(I_{1}\right.$ and $I_{r 1}$ in figure 1$)$ and the second-order scattering $\left(I_{2}\right.$ and $I_{r 2}$ in figure 1).

Light extinction arising during various light paths between the light source and the observer is also computed. To enable this computation, it is assumed that the vertical profiles of the molecular and aerosol concentrations follow exponentially decreasing functions. It is also assumed that aerosol and molecular concentration profiles are uniform over the modeling domain. For further 
details see Aubé et al. (2005).

\subsection{Conversion to astronomical magnitudes}

ILLUMINA was designed to simulate the light scattered back to a spectrometer, so the output is spectral radiance in $\mathrm{Wnm}^{-1} \mathrm{sr}^{-1}$. The model only simulates the contribution of artificial light to the night-sky glow. Contributions from moonlight, stars, the Milky Way, auroras and any other celestial objects are not taken into account.

We aim to do a direct comparison of the model output with the all-sky images taken with an ASTMON instrumentation. The All-Sky Transmission Monitor (ASTMON) is based on a $\mathrm{f}=4.5 \mathrm{~mm}$ fisheye lens and an integrated astronomical CCD camera. It measures the luminance of the all night sky in several wavelength bands. The system is designed to perform a continuous monitoring of the surface brightness of the night sky background in a fully robotic mode. In addition to the sky background brightness, ASTMON can provide atmospheric extinction and cloud coverage estimates for the entire sky surface at the same time (see Aceituno et al. (2011) and Hänel et al. (2017)).

The spectral radiance is not commonly used in astronomical measurements. To compare with ASTMON images we need to convert the output of ILLUMINA to astronomical magnitudes in the visible $B, V, R$ JohnsonCousins filters taking into account the natural brightness of night sky. The Johnson-Cousins photometric system consists in several pass-bands that covers a spectral range from the ultraviolet to the near infrared. For the purposes of this study, only those that covers the visual range will be used: $B, V$ and $R$, which stands for Blue, Visual and Red respectively (see figure 2).

In astronomy, the apparent magnitude $(m)$ of a celestial body is defined from its flux $(F)$, through the expression:

$$
m=m_{r e f}-2.5 \log _{10}\left(\frac{F}{F_{r e f}}\right)
$$

$m_{r e f}$ is the magnitude of a reference source with a flux $F_{r e f}$. The equation above can be used to define the magnitude for a given filter, just considering the total flux in the filter's band.

As equation 1 shows, magnitudes scale is logarithmic. It is defined in such a way that a difference of 5 magnitudes means a factor 100 in flux. On the other hand, a larger flux means a smaller value of the magnitude. 


\begin{tabular}{|c|c|c|}
\hline Johnson Filter & Vega (magnitudes) & Natural Sky (magnitudes/arcsec ${ }^{2}$ ) \\
\hline B & -0.023 & 22.7 \\
V & -0.023 & 21.8 \\
R & -0.023 & 20.9 \\
\hline
\end{tabular}

Table 1: Apparent magnitudes of Vega and natural sky brightness at zenith.

In our case the source will be the artificial light plus natural sky brightness, and the reference object chosen is Vega star, because of its well-known spectrum (Kurucz, 2005). Taking into account that the sky is not a pointlike source, the sky brightness in a given direction is measured in units of magnitudes $/ \operatorname{arcsec}^{2}$, i.e. the magnitude equivalent to the flux received from a region of the sky, in the given direction, which area is equal to $1 \operatorname{arcsec}^{2}$, computed through equation 1 . Table 1 lists the natural night sky (at zenith) and Vega's apparent magnitudes in each filter.

To compute the flux (i.e. artificial light $F_{A L}$ and Vega $F_{r e f}$ ) in each of the filters we define both the flux function (in units of $W / \mathrm{nm}$ ) and the filter transmission function by the same step, multiply them and integrate the result for the filter domain.

Once known Vega's flux in each of the filter's bands we can compute the contribution of the natural night sky $F_{N N S}$ using the magnitudes tabulated in table 1 and using equation 1 in the form:

$$
F_{N N S}=F_{V e g a} 10^{-0.4\left(m_{N N S}-m_{V e g a}\right)}
$$

After converting sky radiance computed by ILLUMINA to flux in each filter, we finally add it to $F_{N N S}$ in order to compare the results with measurements with ASTMON instrumentation:

$$
m=m_{V e g a}-2.5 \log _{10}\left(\frac{F_{A L}+F_{N N S}}{F_{V e g a}}\right)
$$

\section{Case of study: Parc Astronomic Montsec}

Parc Astronomic Montsec (PAM) is located in the north-east of Spain (figure 3). Scientific studies certified this location as ideal for astronomic purposes due to its low level of rainfall and humidity, the high ratio of clear nights and its elevation $(1600 \mathrm{~m}$ above sea level). 


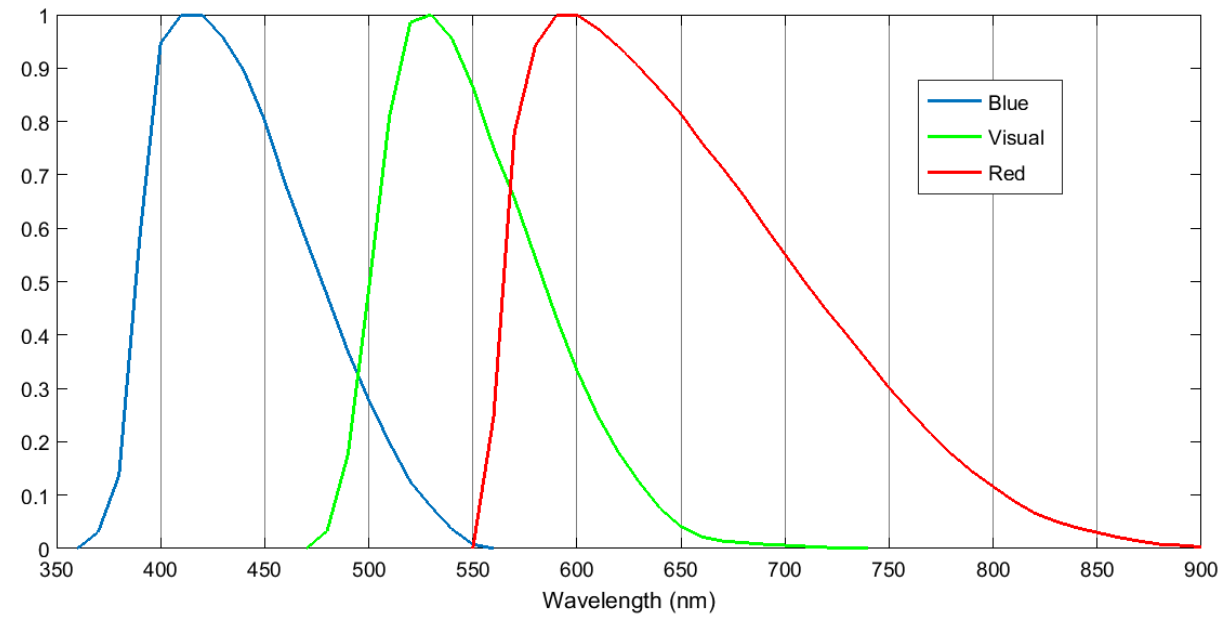

Figure 2: Normalized transmittances of the $B, V, R$ Johnson-Cousins filters.

The sky above the PAM is considered as free from light pollution and hence it is protected by the local legal framework. Only some bright sky areas close to the horizon are detected. However, there are some cities and towns around the PAM that are actually affecting astronomical measurements.

In particular Lleida, the biggest city in the area, is thought to be one of the major stakeholders polluting the sky above PAM. It is a middle-sized city with almost 140,000 inhabitants, with an extension of approximately 220 $\mathrm{km}^{2}$, located at $47 \mathrm{~km}$ around 190 in azimuth ( $0^{\circ}$ pointing to the North). Its lighting system is affecting the measurements taken from the PAM in lines of sight close to its location and its sky-glow (defined as the dome of light over a city) is visible to the naked eye from the observatory, as shown in figure 4.

Beyond the significance of studying how the city is affecting the sky over the observatory, it is relevant due to an update of the lighting systems of the city that took place in 2014. This allows to study the resulting modifications in sky brightness and validate the model in both scenarios.

We had access to an accurate inventory of public light systems before and after 2014 courtesy by the city council of Lleida and the company Citelum. The information provided for each light source included: consumed power, kind and height of light source, percentage of light emitted upwards and location in coordinates. 

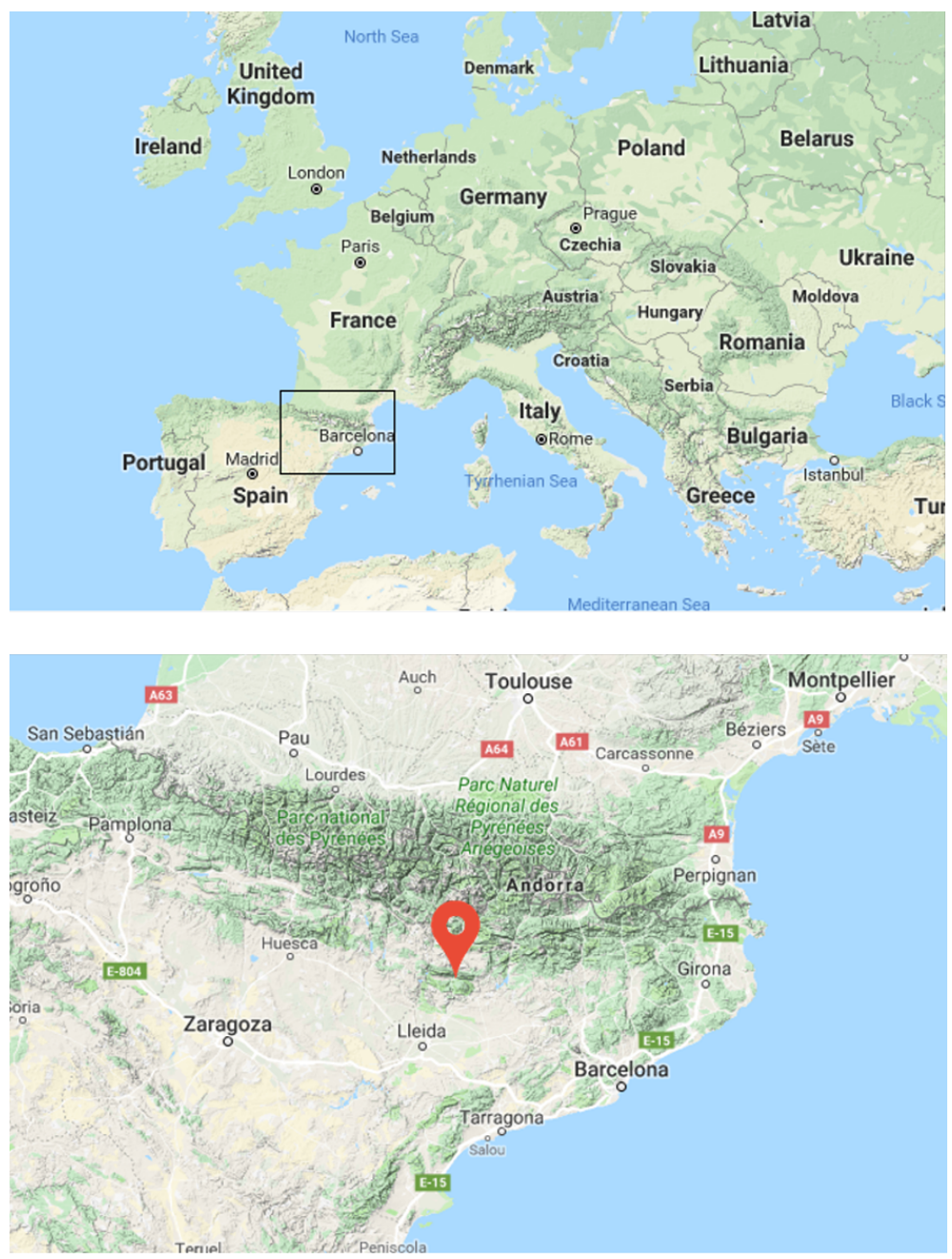

Figure 3: Location of the Parc Astronòmic Montsec. 


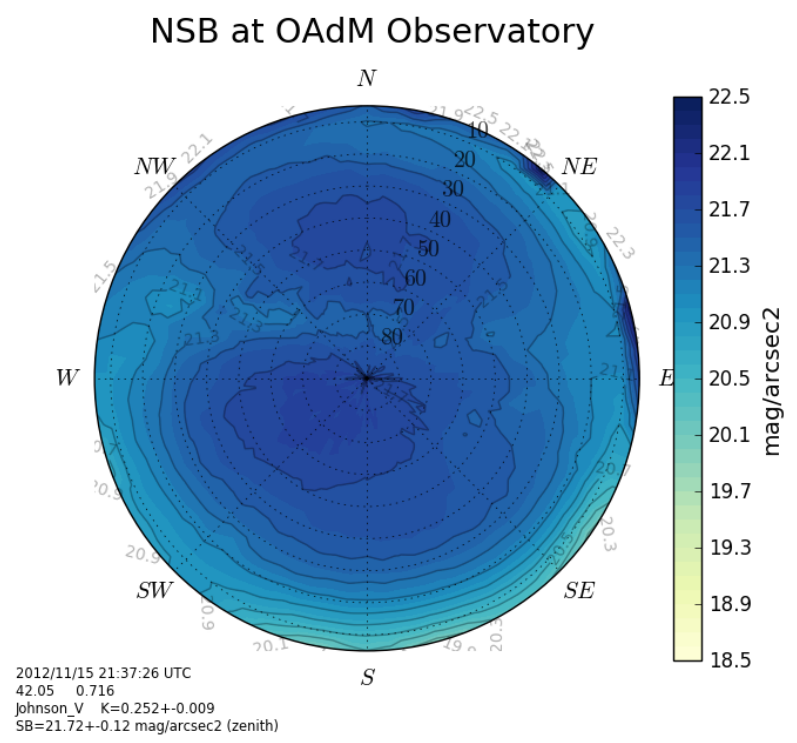

Figure 4: All-sky map over the PAM in V filter. Measurements taken with ASTMON instrumentation (All-Sky Transmition MONitor).

In order to define Lleida as a light source in the way ILLUMINA requires we computed a 3D plot to know how the emitted power is distributed over the city. As can be seen in figure 5 there is a central zone where most of the power emitted is concentrated and two small concentrations far from the biggest one. A more detailed analysis shows that $97.5 \%$ of the emitted power is concentrated in a radius of $6 \mathrm{~km}$. Therefore, we adopt this radius as the size of the ILLUMINA light source zone.

\subsection{Inventory of lights}

There are several kind of lighting systems in both 2013 and 2015 inventories of Lleida. However, in each case there are 3 kind of lamps responsible for more than $95 \%$ of the emitted power (table 2). It is accurate enough though just to take into account the three dominant lamps in each case.

The update of the lighting system consisted mostly in replacing all the mercury vapor (MV) and some of the metal halide (MH) lamps by LEDs $(4000 \mathrm{~K})$. The total number of high pressure sodium vapor (HPSV) is the same in both inventories, the reduction in the percentage is due to a higher number of total lamps. Although the total number of light sources increased 


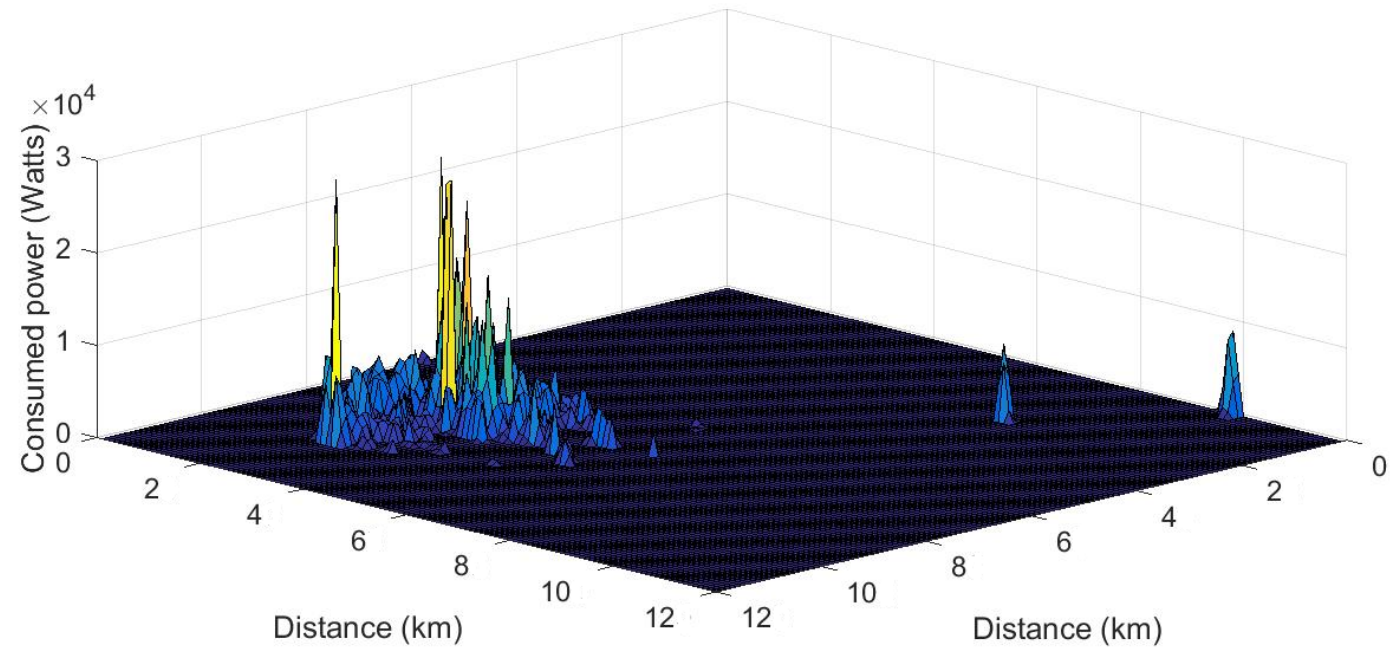

Figure 5: Light power emission in Lleida vs location.

\begin{tabular}{|c|c|c|c|c|c|c|c|}
\hline & $\begin{array}{c}\text { HPSV } \\
(\%)\end{array}$ & $\begin{array}{c}\text { MH } \\
(\%)\end{array}$ & $\begin{array}{c}\text { LED 4000K } \\
(\%)\end{array}$ & $\begin{array}{c}\text { MV } \\
(\%)\end{array}$ & $\begin{array}{c}\text { Other } \\
(\%)\end{array}$ & \# Lamps & $\begin{array}{c}\text { Total Power } \\
(\mathrm{kW})\end{array}$ \\
\hline 2015 & 65 & 16 & 15 & 0 & 4 & 25208 & 1934.4 \\
2013 & 71 & 19 & 0 & 7 & 3 & 23004 & 3006.4 \\
\hline
\end{tabular}

Table 2: Lleida inventory of lights. HPSV: High Pressure Sodium Vapor, MH: Metal Halide, MV: Mercury Vapor.

with the update, the power emission diminished due to the lower average emitted power of the lamps installed. The 2015 inventory has shown a total emitted power reduction of $33 \%$.

\subsection{Model parameters}

Besides the inventory of lights, ILLUMINA needs a set of parameters to perform the computation: satellite data, wavelength domain, atmosphere definition, region dimension and lines of sight of interest.

The satellite data is used to determine several model parameters. VIIRSDNB data is used to compute the light emitted by each region $(1 \mathrm{x} 1 \mathrm{~km}$ that is a pixel in the image). MODIS imagery provides the ground reflectance. Finally, SRTM data allows to create a digital elevation model of the area. We 
have used satellite images from April in both cases (2013 and 2015) because usually in this month Lleida area is free of fog and other phenomena that could alter the data.

The wavelength range defined has its minimum at $350 \mathrm{~nm}$ and a maximum at $830 \mathrm{~nm}$, and it is divided in 16 bins of $30 \mathrm{~nm}$.

The atmosphere characterization is identical for both scenarios (2013 and 2015) in order to ensure that the results obeys only to the different light sources. It has been assumed a rural environment with a relative humidity of $70 \%$, atmospheric standard pressure $(101,3 \mathrm{kPa}$ at sea level) and clear sky (no clouds). Typical aerosol optical depth (AOD) and angstrom coefficients $(\mathrm{AC})$ are taken from the data from AERONET (AErosol RObotic NETwork, Holben et al. (1998)) for April 2013: AOD 0.074 and AC 0.977.

The dimensions of the region of study are of $466 \times 377 \mathrm{~km}$ (figure 6). The sky mapping goes from $5^{\circ}$ to $90^{\circ}$ in elevation with steps of $5^{\circ}$, and covers all the azimuth range with steps of $5^{\circ}$ too. This grid results in 1225 lines of sight.
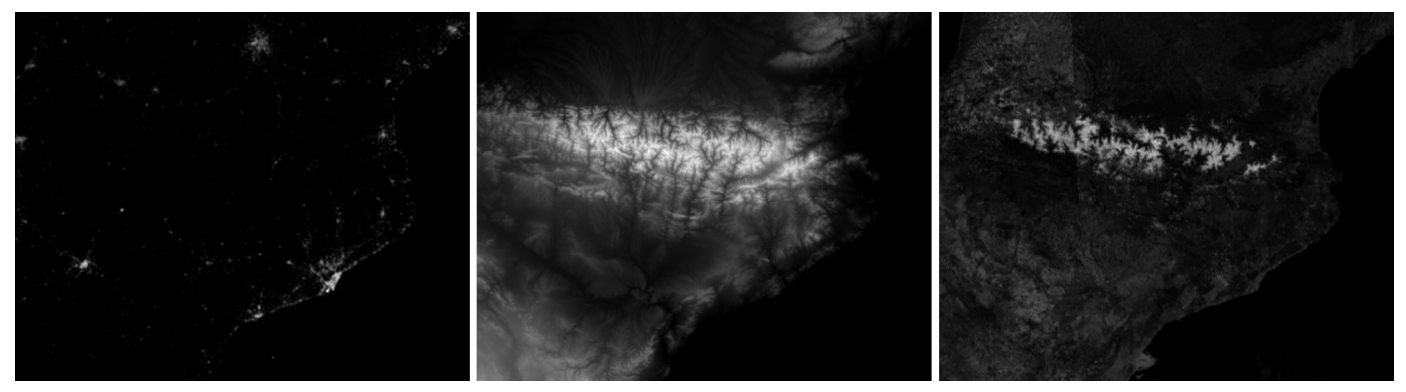

Figure 6: Ground reflectance satellite image of project framework.

\section{Results}

The first set of figures in this section (figure 7) show all-sky ASTMON images in the Johnson-Cousins filters $B, V$ and $R$ taken in November 2012 and May 2015. They are used to check the ILLUMINA model. All the images represent the sky from the PAM, with the azimuth's origin pointing to the North. The elevation starts at $5^{\circ}$ in the outer radius and increases as the radius shortens, with the zenith $\left(h=90^{\circ}\right)$ at the center. 
Figures 8 to 13 show all-sky results from ILLUMINA for both 2013 and 2015 scenarios. A comparison done by subtracting 2013 magnitudes to 2015 results is also shown. A positive value means that 2015 is fainter than 2013.

We represent also the differences between the $(B-V)$ and $(V-R)$ color index for both scenarios (figures 14 and 15). A color index is defined as the difference between the magnitudes in two filters. Colors index help to analyze the variation of the spectral distribution of the sky brightness. For instance, the bluer the sky, the smaller $(B-V)$ value (remember that the value of the magnitude decreases as the flux increases).

To see in detail the relation between light sources and spectral behaviour of artificial light we have compared the spectral radiance (in $W n m^{-1} s r^{-1}$ ) emitted in each of the 16 spectral bins in 2013 and 2015. To do so the only variable parameter is the light source inventory. All other parameter is fixed for both cases. The figures 16 and 17 just show the azimuth angle of interest where Lleida is located $\left(145^{\circ}-235^{\circ}\right)$. The equation used for the comparison is:

$$
C=\frac{F_{2015}-F_{2013}}{F_{2013}}
$$

$F_{2015}$ and $F_{2013}$ are the spectral radiance obtained with 2015 and 2013 lighting infrastructure respectively. Positive values mean that 2015 light sources emit more than in 2013 and negative values the opposite.

\section{Discussion}

When comparing ILLUMINA results with ASTMON data, we need to take into account that, as seen in figure 4, Lleida is not the only source of light pollution. There are other directions that are affected such as $120^{\circ}$, which coincides with the direction to Barcelona, and $160^{\circ}$ that points to Balaguer, a town smaller than Lleida but much closer. Furthermore, other elements can affect the measurement, such as the presence of the Milky Way. This makes difficult to perform an accurate comparison between the model and the measured all-sky ASTMON data for every line of sight.

Figures 8-10-12 show that, as expected, the most affected lines of sight are in the direction of Lleida, $175-205^{\circ}$ in azimuth. It is also obvious that the effect is more pronounce for low elevation angles, up to $20^{\circ}$, in good agreement 

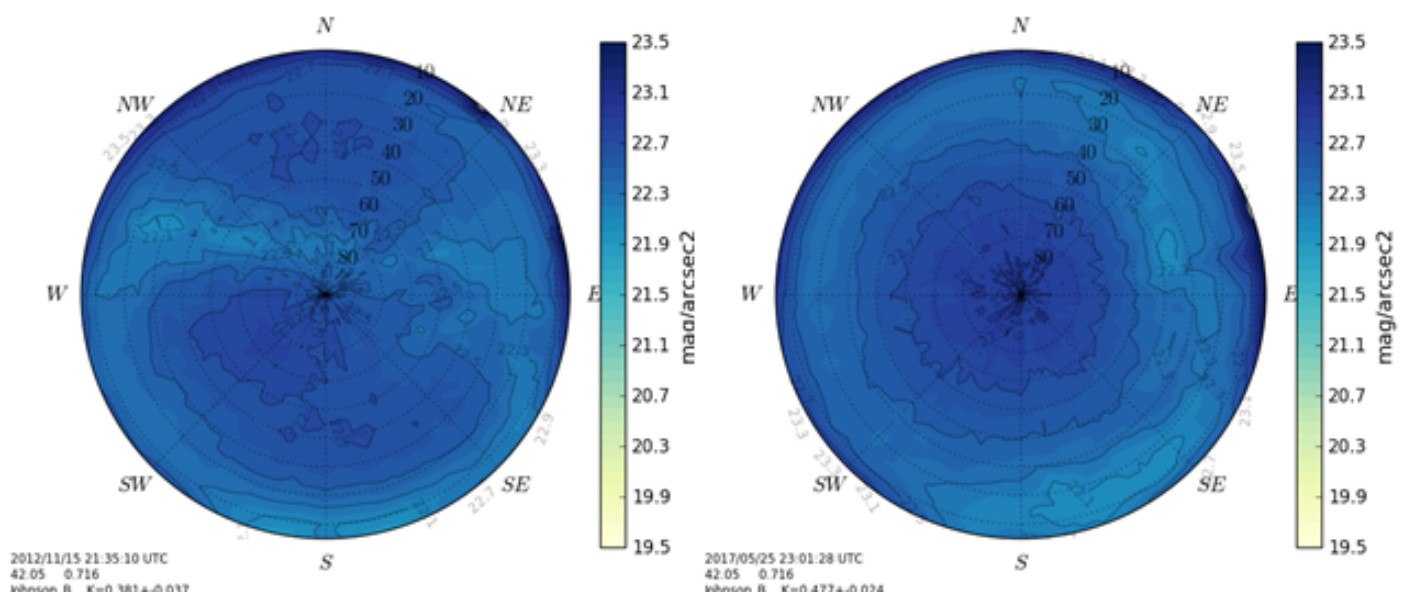

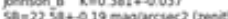
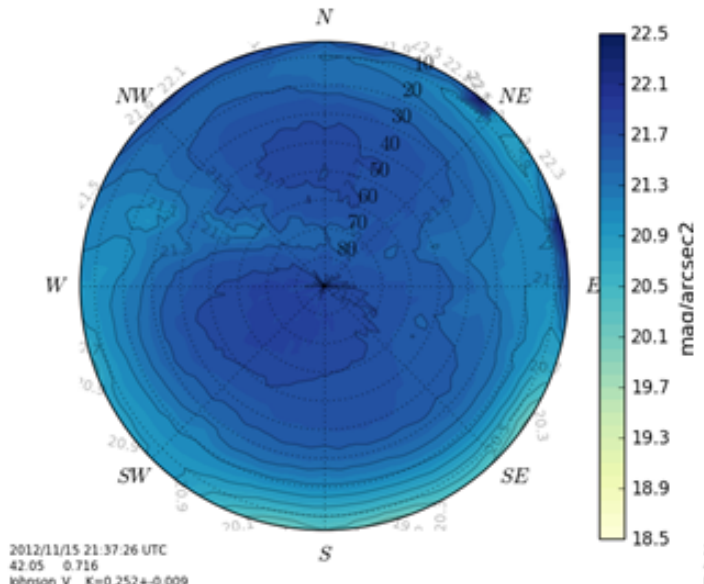

thesh $8=0.477+0.024$

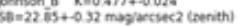

panion V $x=0.252+0.000$
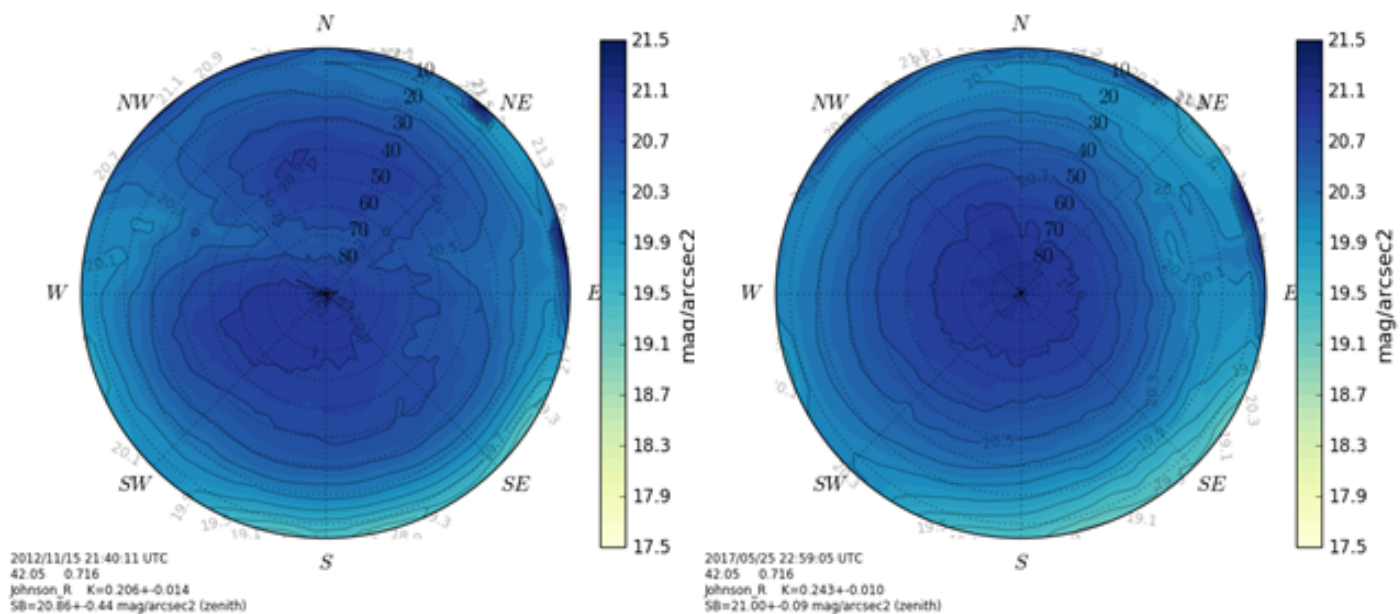

Figure 7: All-sky ASTMON maps. Left: 11-2012. Right: 05-2017. Top: B filter. Middle: V filter. Bottom: R filter. 

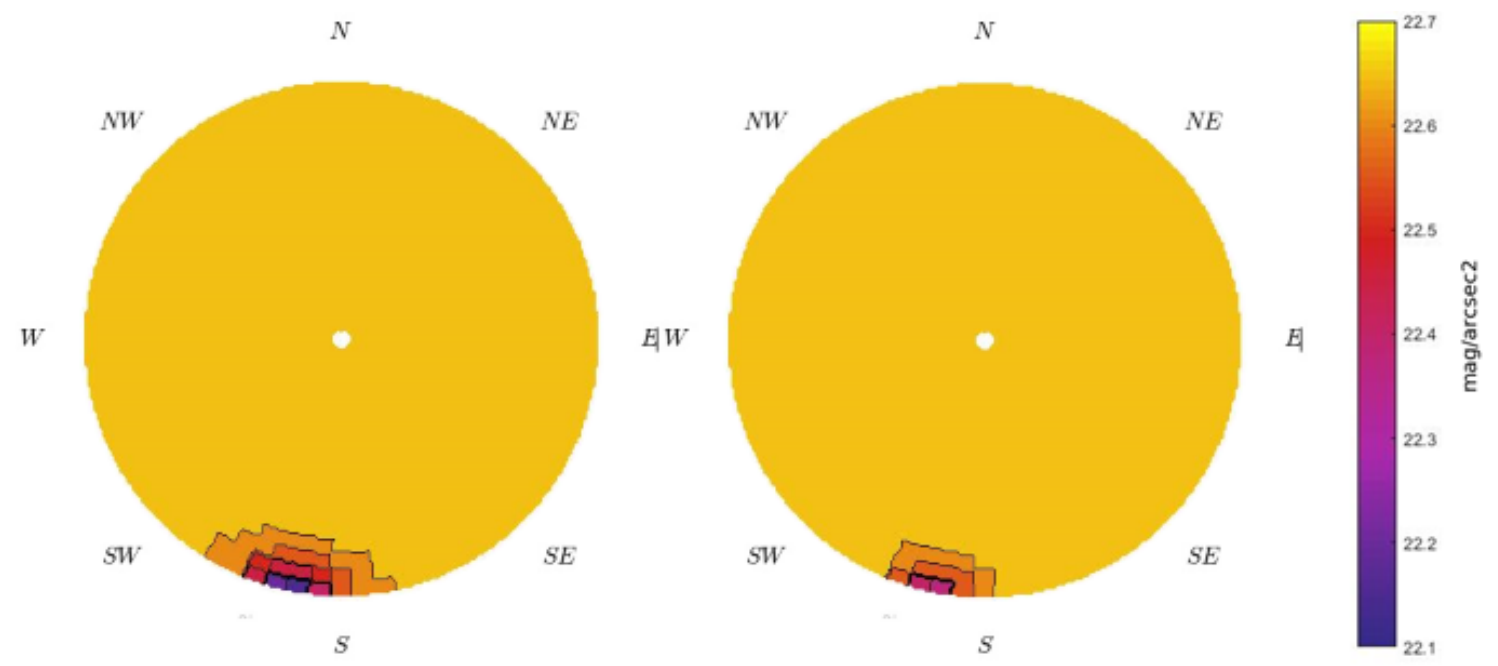

Figure 8: All-sky maps obtained by the model for B filter. Left: 2013. Right: 2015.

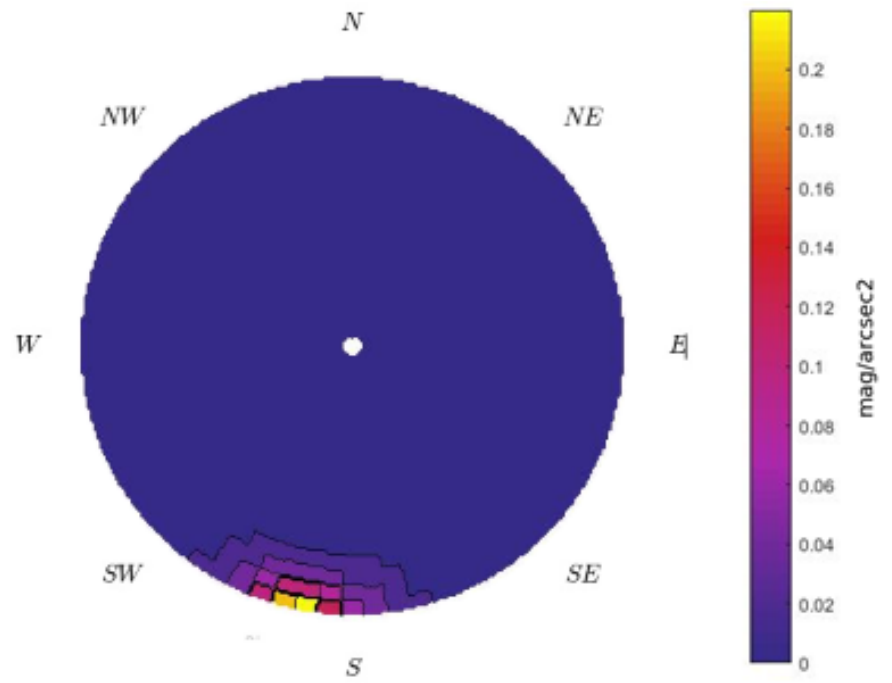

Figure 9: Received flux in $B$ filter comparison between 2015 and 2013. The data shown is the result of subtracting the data from 2013 to the data from 2015. 
$N$

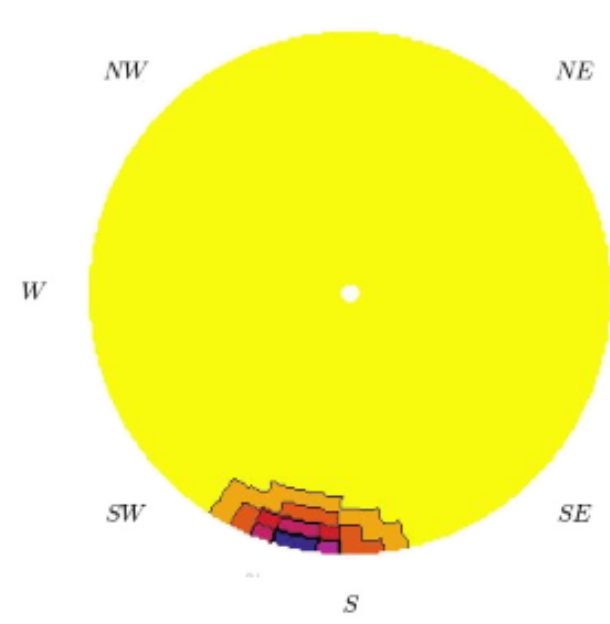

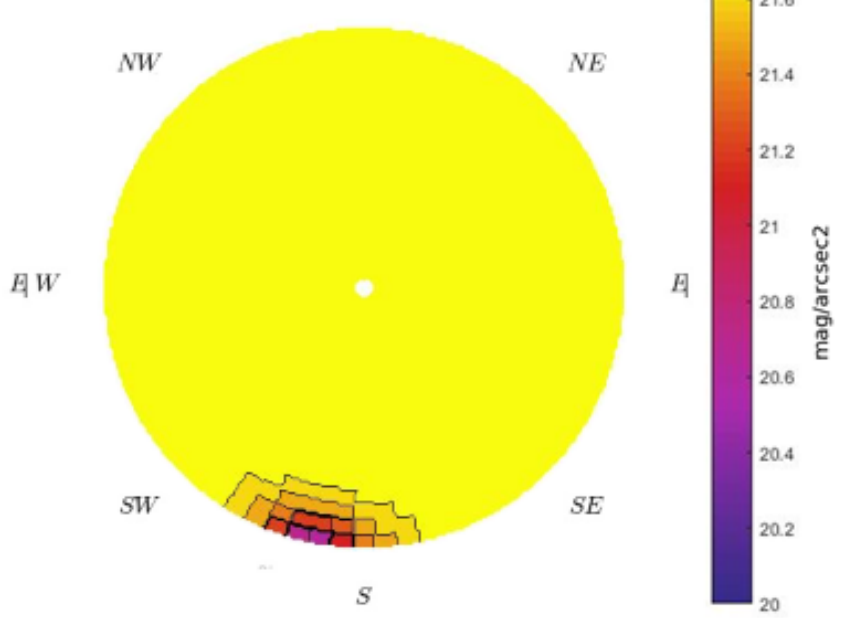

Figure 10: All-sky maps obtained by the model for $V$ filter. Left: 2013. Right: 2015.

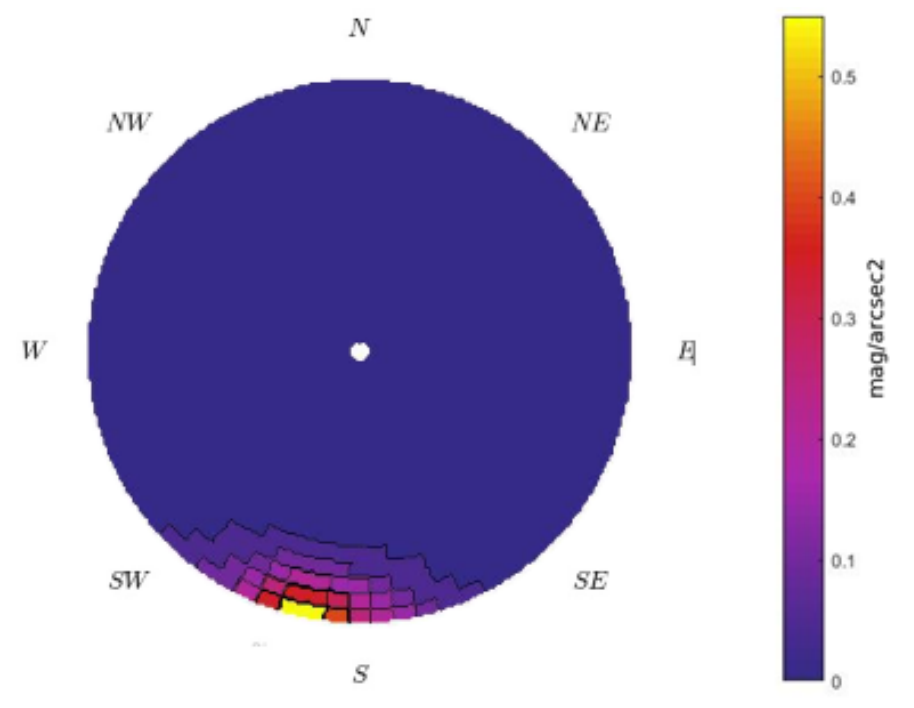

Figure 11: Received flux in $V$ filter comparison between 2015 and 2013. The data shown is the result of subtracting the data from 2013 to the data from 2015. 

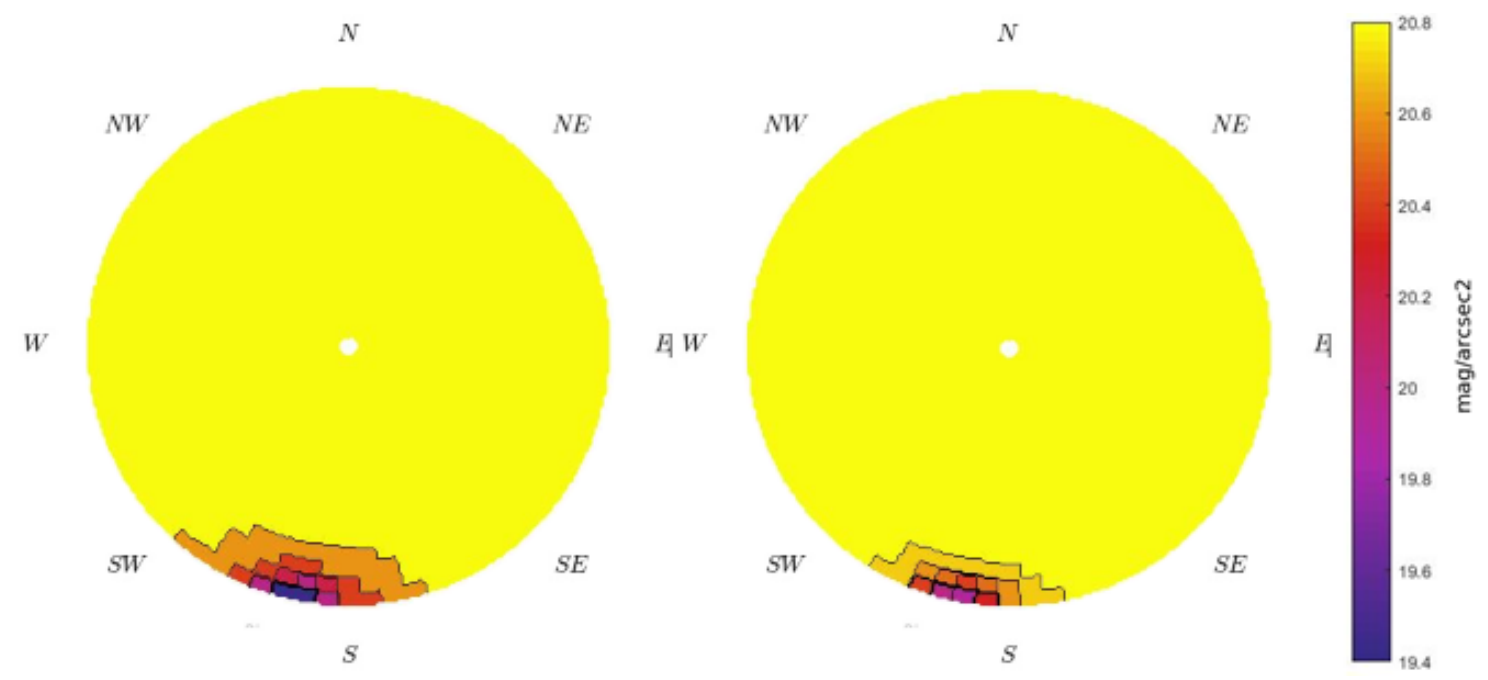

Figure 12: All-sky maps obtained by the model for $R$ filter. Left: 2013. Right: 2015.

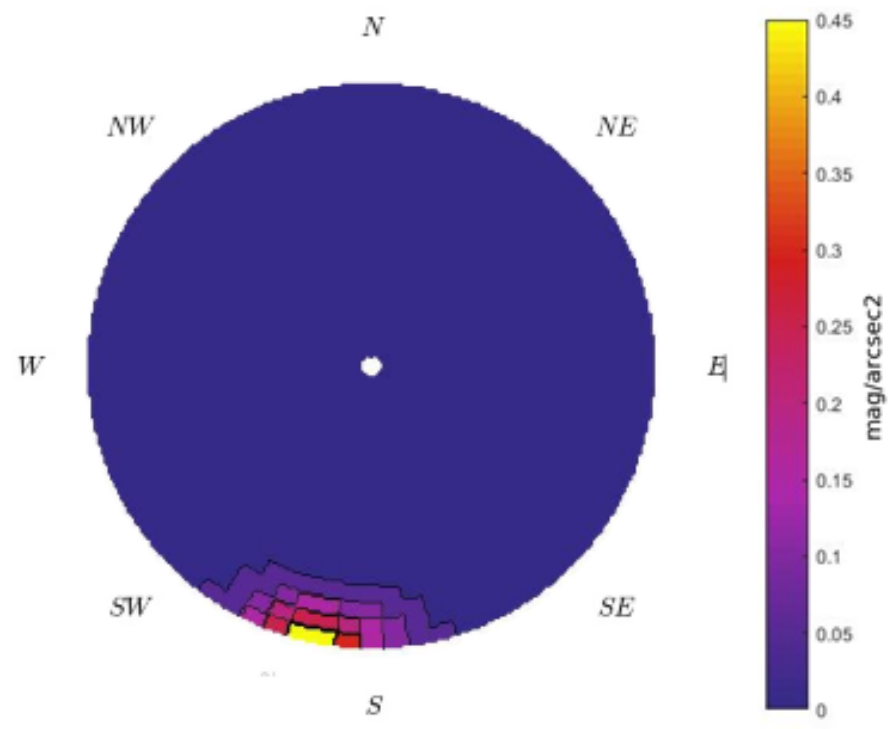

Figure 13: Received flux in $R$ filter comparison between 2015 and 2013. The data shown is the result of subtracting the data from 2013 to the data from 2015. 


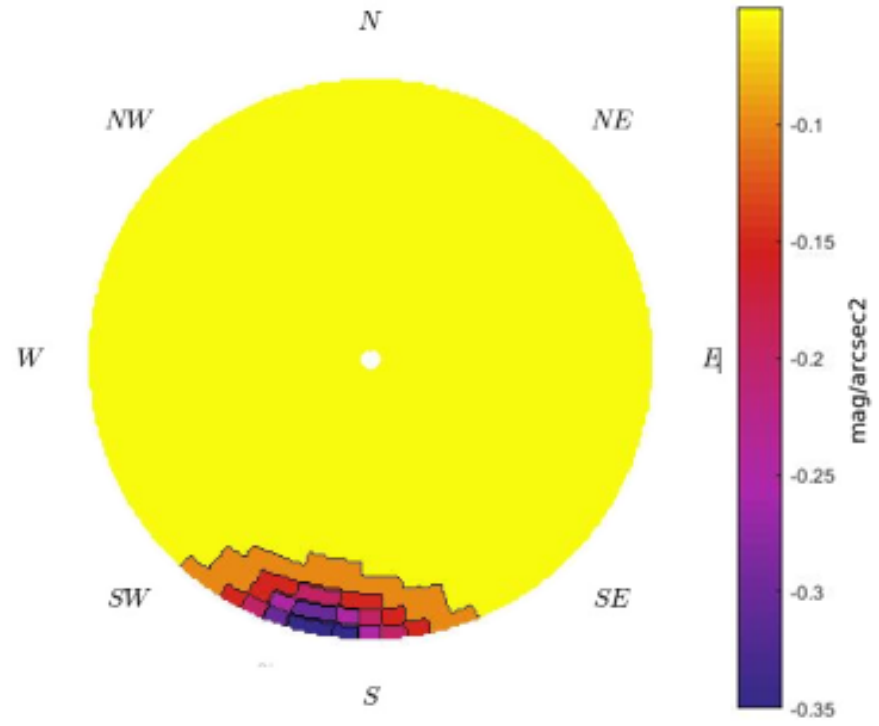

Figure 14: All-sky difference map of sky brigthnes in B and V between 2015 and 2013 (2015-2013).

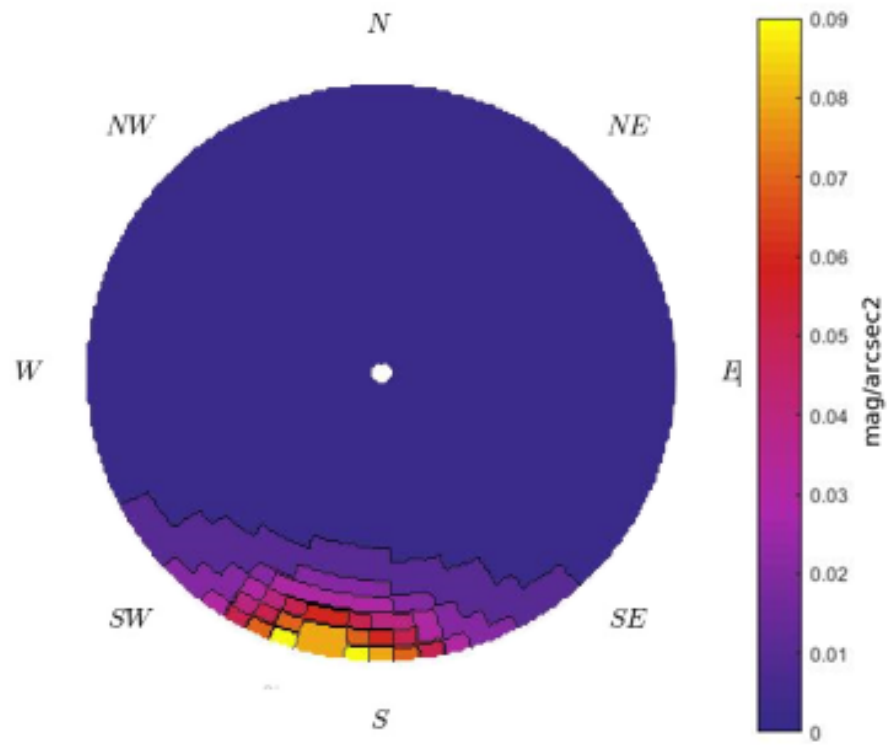

Figure 15: All-sky difference map of sky brigthnes in V and R between 2015 and 2013 (2015-2013). 

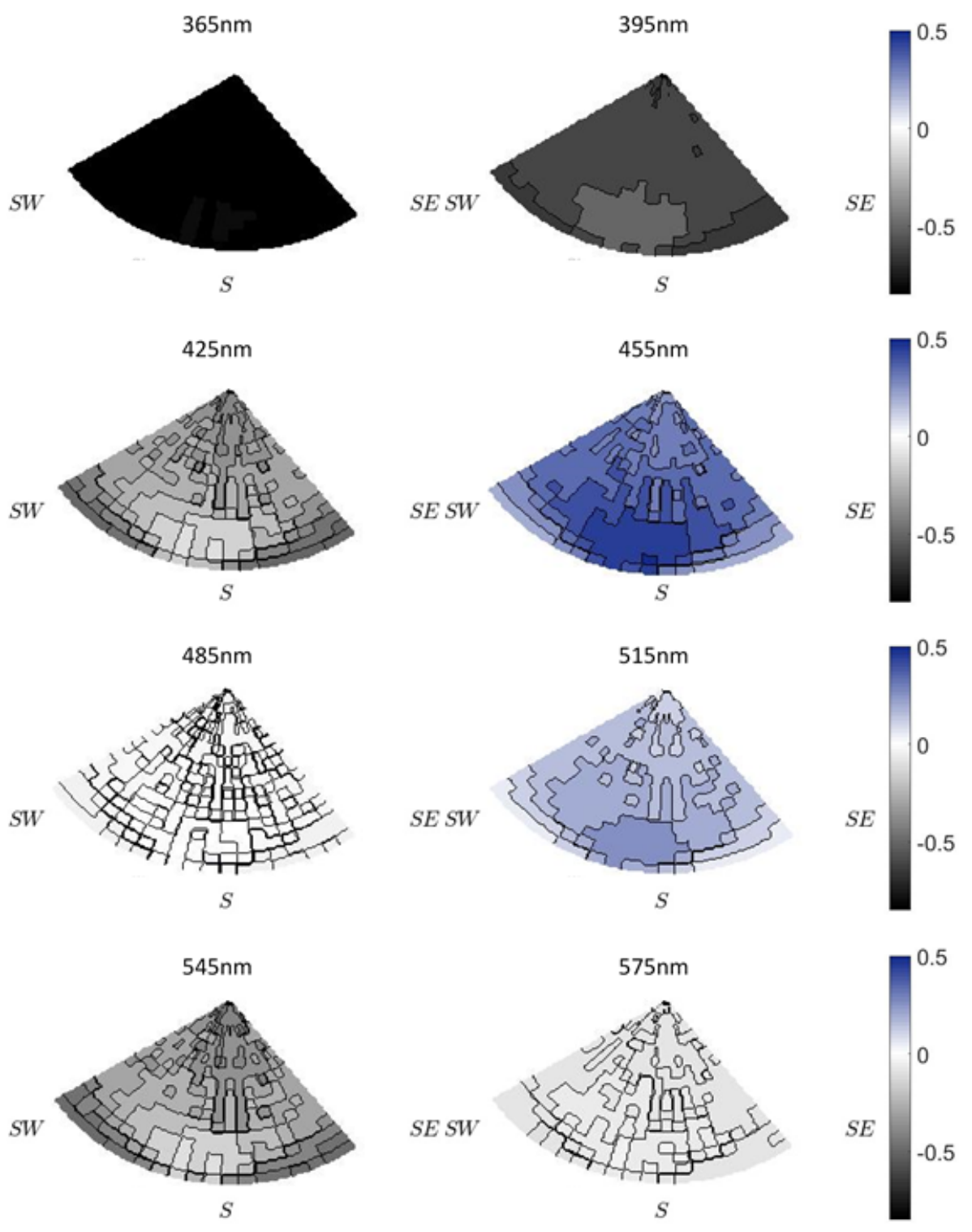

Figure 16: Comparison of emitted flux by Lleida in several spectral ranges between 2013 and 2015, only taking into account the different spectral behaviour of the lights. The comparison follows the expression: $C=\frac{F_{2015}-F_{2013}}{F_{2013}}$ 


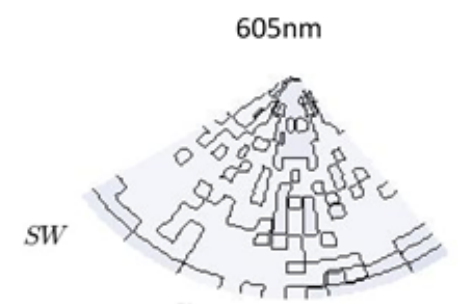

$S$
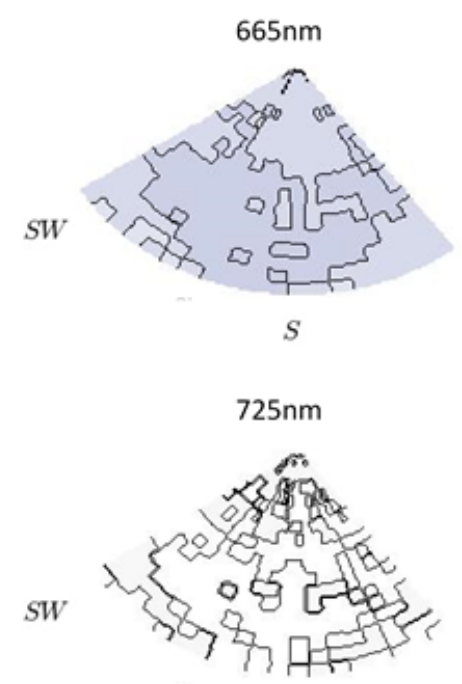

$S$

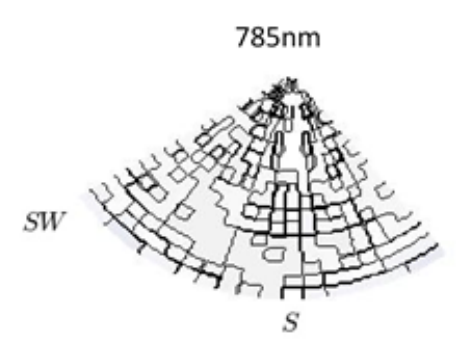

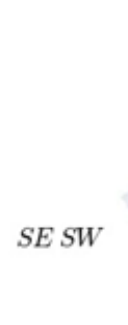

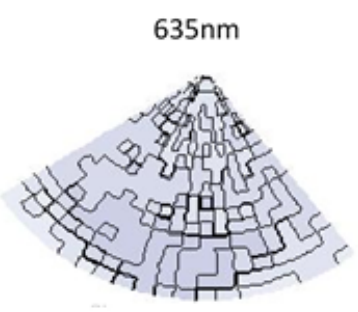

S
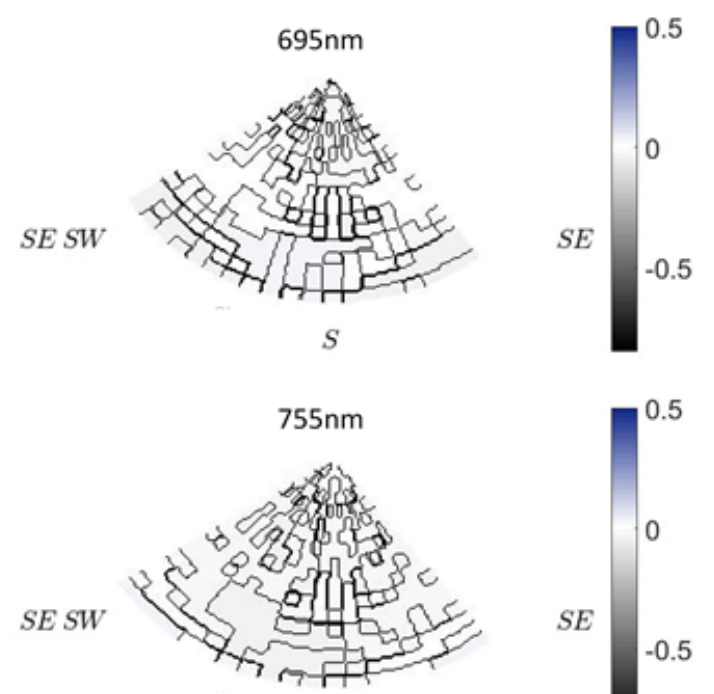

S

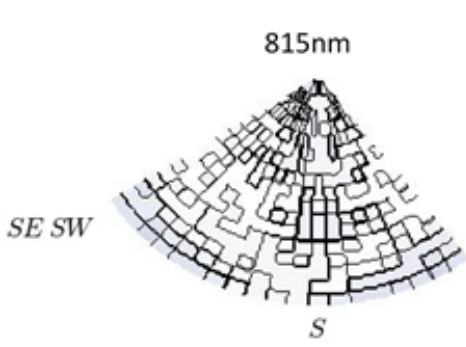

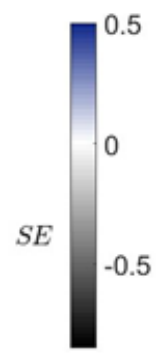
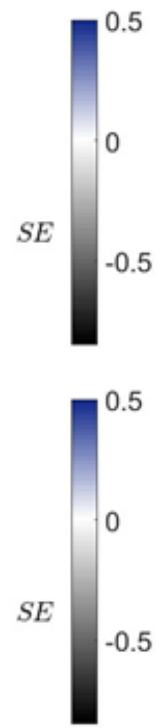

Figure 17: Comparison of emitted flux by Lleida in several spectral ranges between 2013 and 2015, only taking into account the different spectral behaviour of the lights. The comparison follows the expression: $C=\frac{F_{2015}-F_{2013}}{F_{2013}}$ 
with the ASTMON images. Measured and computed light pollution in the direction of Lleida at low elevation, differ only slightly and never more than 0.1 magnitudes (that is a variation of $9 \%$ in flux between measured and computed).

On the other hand, images from model show that light pollution is not uniform among the filters used. Images from 2013 show that in B there is a brightening of $0.6 \mathrm{mag}$ with respect natural sky brightness (from 22.7 to 22.1 ), in $\mathrm{V}$ of $1.7 \mathrm{mag}$ (from 21.7 to 20.0) and in $\mathrm{R}$ of $1.4 \mathrm{mag}$ (from 20.8 to 19.4). To see it in a linear scale (magnitudes are logarithmic) in 2013 the sky over the PAM in the direction of Lleida was brightened 0.75 times in B, 3.78 times in $\mathrm{V}$ and 2.62 times in $\mathrm{R}$ due to Lleida skyglow.

Direct comparison images show a clear reduction of the sky brightness due to Lleida over the PAM in the 2015. The reduction is not uniform in different spectral ranges. In $B$ it is around 0.2 magnitudes (17\% of flux) at maximum, and around $0.5(37 \%)$ in the other filters. This is thought to be a result of the shift of the spectrum emitted to shorter wavelengths due to the installation of $4000 \mathrm{~K}$ LEDs. This uneven reduction affects the color $B-V$ index in particular.

This effect can be analyzed in more detail with help of figures 16 and 17 . They show the comparison between spectral fluxes, a negative number means that the old lights emit more than the new ones and vice-versa. Up to $425 \mathrm{~nm}$ old lights emit much more due to emission of mercury vapor lights present only in the old inventory. At $455 \mathrm{~nm}$ and $515 \mathrm{~nm}$ there is much more emission with the new lights (up to $50 \%$ more) linked with the emission of LED lights of $4000 \mathrm{~K}$. There is a little more emission of the old lights at $545 \mathrm{~nm}$ due to a peak in the spectrum of mercury vapor, removed in the 2015. At larger wavelength there are barely no differences, due to the emission is dominated by high pressure sodium vapor and metal halide lights that are present in both inventories.

As a summary, the model show that, with the new lighting systems, Lleida has reduced the total amount of emitted power in every filter, but it has increased the shorter wavelengths relative contribution due to the introduction of 4000K LEDs.

\section{Conclusions}

The methodology presented in this work shows to be suitable to study the contribution of artificial light sources to the sky brightness. In our case 
of study, Lleida has been detected in the expected azimuth and elevation. Lleida enlightens the sky of Montsec, but the light pollution is confined in a set of lines of sight around $190^{\circ}$ in azimuth and up to $25^{\circ}$ in elevation. In spite of simplifications as not taking into account natural sources of light, such as the Milky Way, and other cities around the observer, the results in the lines of sights affected by Lleida are in good agreement with measurements.

This study shows also that Lleida is affecting the measurements of the night-sky made from the PAM in the whole spectrum. The light system change that took place in 2014 altered the light emission of Lleida, mostly reducing the total flux emitted in every filter and shifting some emission from the $V$ filter to the $B$ one (i.e. to shorter wavelengths). As a matter of fact we observed a remarkable change in color index $B-V$, as it was pointed out by observers in the PAM.

When isolating the spectral behavior (figure 16 and 17) we observe reductions up to a factor 0.8 in very short wavelengths, from 365 to $425 \mathrm{~nm}$, and increments up to 0.5 at 455 and close to 0.3 at $515 \mathrm{~nm}$, a wavelength close to the peak of human scotopic sensitivity. This findings are directly related with the emission spectrum of the light sources present in 2013 and 2015.

It means that a reduction of the total luminous flux emitted by light sources does not necessarily means a reduction in the amount of luminous flux emitted in each wavelength. Even if it seems to the naked eye that there has been a reduction in emission it could have just only shifted to wavelengths not as sensitive to the eye.

This methodology can be used easily as a first overall estimation of sky brightness changes even with relatively low accurate inventory of lights and conditions. It could be useful for cities and towns to help in choosing properly the lighting system to be installed or replaced to reduce their contribution to light pollution. To perform more specific studies in which certain wavelength must be studied carefully the user have to bear in mind that it is required to obtain an almost perfect spectrum of the lights studied.

This project was a first trial working with ILLUMINA, in the light of the results obtained and the offered collaboration from institutions it could be a nice starting point for studying other areas of interest. As a matter of fact this methodology will be used in the international and UE financed project "Pyrénées la nuit" to model the night sky at least in 3 different locations within the Pyrenees. 


\section{Acknowledgments}

M. A. thanks the Fonds de recherche du Québec - Nature et technologies (FRQNT) for financial support through the Research program for college researchers.

\section{Bibliography}

Aceituno, J., Sánchez, S., Aceituno, F., Galadí-Enríquez, D., Negro, J., Soriguer, R., Gomez, G. S., 2011. An all-sky transmission monitor: Astmon. Publications of the Astronomical Society of the Pacific 123 (907), 1076.

Aubé, M., 2007. Light pollution modeling and detection in a heterogeneous environment. In: Proceedings of Starlight 2007 conference, La Palma, Spain.

Aubé, M., Franchomme-Fossé, L., Robert-Staehler, P., Houle, V., 2005. Light pollution modelling and detection in a heterogeneous environment: toward a night-time aerosol optical depth retreival method. In: Atmospheric and Environmental Remote Sensing Data Processing and Utilization: Numerical Atmospheric Prediction and Environmental Monitoring. Vol. 5890. International Society for Optics and Photonics, p. 589012.

Aubé, M., Roby, J., 2014. Sky brightness levels before and after the creation of the first international dark sky reserve, mont-mégantic observatory, québec, canada. Journal of Quantitative Spectroscopy and Radiative Transfer 139, 52-63.

Berry, R. L., 1976. Light pollution in southern ontario. Journal of the Royal Astronomical Society of Canada 70, 97.

Bertiau, F., de Graeve, E., Treanor, P. J., 1973. The artificial night-sky illumination in italy. Vatican Observatory Publications 1, 159-179.

Cousins, A., 1976. Standard stars for vri photometry with s25 response photocathodes. Monthly Notes of the Astronomical Society of South Africa 35,70 . 
Hänel, A., Posch, T., Ribas, S. J., Aubé, M., Duriscoe, D., Jechow, A., Kollath, Z., Lolkema, D. E., Moore, C., Schmidt, N., et al., 2017. Measuring night sky brightness: methods and challenges. Journal of Quantitative Spectroscopy and Radiative Transfer.

Holben, B. N., Eck, T. F., Slutsker, I., Tanre, D., Buis, J., Setzer, A., Vermote, E., Reagan, J., Kaufman, Y., Nakajima, T., et al., 1998. Aeroneta federated instrument network and data archive for aerosol characterization. Remote sensing of environment 66 (1), 1-16.

Johnson, H., Morgan, W., 1953. Fundamental stellar photometry for standards of spectral type on the revised system of the yerkes spectral atlas. The Astrophysical Journal 117, 313.

Johnson, H., Morgan, W., 1955. The ubv photometric system. Ann. Astrophys 18, 292-296.

Kocifaj, M., 2007. Light-pollution model for cloudy and cloudless night skies with ground-based light sources. Applied optics 46 (15), 3013-3022.

Kurucz, R. L., 2005. Atlas12, synthe, atlas9, width9, et cetera. Memorie della Societa Astronomica Italiana Supplementi 8, 14.

Normandin, M., 1974. Appendix d in demande d'octroi au conseil national de recherches du canada pour l'installation d'un observatoire astronomique au quebec.

Ribas, S. J., 2016. Caracteritzaci de la contaminaci lumnica en zones protegides i urbanes. Ph.D. thesis.

Ribas, S. J., Canal-Domingo, R., 2015. Seguiment de la qualitat del cel nocturn del territori starlight del montsec i zones veïnes.

Riegel, K. W., 1973. Light pollution: outdoor lighting is a growing threat to astronomy. Science 179 (4080), 1285-1291.

Treanor, P. J., 1973. A simple propagation law for artificial night-sky illumination. The Observatory 93, 117-120.

Walker, M. F., 1970. The california site survey. Publications of the Astronomical Society of the Pacific 82 (487), 672. 
Walker, M. F., 1973. Light pollution in california and arizona. Publications of the Astronomical Society of the Pacific 85 (507), 508.

Walker, M. F., 1977. The effects of urban lighting on the brightness of the night sky. Publications of the Astronomical Society of the Pacific 89 (529), 405 . 